\title{
Dioscin attenuates lipopolysaccharide-induced inflammatory myocardial injury through oxidative stress-related pathway
}

\author{
Zheng $\mathrm{Xu}^{1 *}$, Xiaomin $\mathrm{Li}^{2+}$, Xuewen $\mathrm{Li}^{1}$, Yuping Gao ${ }^{1}$, Xiaolong $\mathrm{Mi}^{1}$ \\ ${ }^{1}$ Department of Cardiovascular Medicine, Shanxi Bethune Hospital, Third Clinical Medical College of Shanxi Medical University, Taiyuan, China; \\ ${ }^{2}$ Department of General Surgery, Shanxi Bethune Hospital, Third Clinical Medical College of Shanxi Medical University, Taiyuan, China \\ Contributions: (I) Conception and design: Z Xu; (II) Administrative support: Z Xu; (III) Provision of study materials or patients: Xuewen Li, Y Gao; \\ (IV) Collection and assembly of data: X Mi; (V) Data analysis and interpretation: Z Xu, Xiaomin Li; (VI) Manuscript writing: All authors; (VII) Final \\ approval of manuscript: All authors. \\ "These authors contributed equally to this work as co-first author. \\ Correspondence to: Zheng Xu. Department of Cardiovascular Medicine, Shanxi Bethune Hospital, Third Clinical Medical College of Shanxi Medical \\ University, Taiyuan, China. Email: xuzheng5808@126.com.
}

\begin{abstract}
Background Lipopolysaccharide (LPS) is one of the main causes of myocardial injury. Dioscin has a protective effect on myocardial injury induced by LPS; however, the biological function and mechanism remain unclear. The purpose of this study was to investigate the effect of dioscin on myocardial injury induced by LPS.
\end{abstract}

Methods: The myocardial injury model was constructed through LPS treatment of primary rat cardiomyocytes. Cardiomyocytes were treated with different concentrations of dioscin (50, 100, and $200 \mathrm{ng} / \mathrm{mL}$ ). MTT was used to detect the activity of cardiomyocytes; flow cytometry and TUNEL assay were used to detect apoptosis; and enzyme-linked immunosorbent assay (ELISA) was used to detect the levels of inflammatory cytokines, tumor necrosis factor- $\alpha$ (TNF- $\alpha$ ), interleukin-1 $\beta$ (IL-1 $\beta$ ), and interleukin-6 (IL-6). The release of superoxide dismutase (SOD), malondialdehyde (MDA), and glutathione (GSH) was detected according to the kit instructions. The levels of apoptosis-related proteins (Bax, caspase-3, and Bcl 2) and the Nrf2-Keap1 pathway proteins were detected by western blot.

Results: Dioscin significantly reduced LPS-induced cardiomyocyte injury in neonatal rats in a concentration- and time-dependent manner. Dioscin also significantly inhibited cardiomyocyte inflammation and apoptosis induced by LPS. With the increase of dioscin concentration, reactive oxygen species (ROS) and MDA were downregulated, and SOD and GSH were upregulated. Moreover, dioscin inhibited LPSinduced myocardial injury by inhibiting the Nrf2-Keap1 pathway.

Conclusions: Our study suggests that dioscin attenuates LPS-induced myocardial injury through oxidative stress-related pathways.

Keywords: Dioscin; oxidative stress; lipopolysaccharide-induced myocardial injury (LPS-induced myocardial injury); inflammation; apoptosis

Submitted May 25, 2021. Accepted for publication Jul 08, 2021.

doi: $10.21037 /$ apm-21-1613

View this article at: https://dx.doi.org/10.21037/apm-21-1613

\section{Introduction}

Myocarditis is a myocardial inflammatory disease which can lead to cardiac dysfunction, reduce systolic and diastolic function, and cause arrhythmia. Myocarditis can result from a variety of causes, including infection, allergic reaction, and immune-related injury (1). At present, the pathogenesis of myocarditis is not fully known. Therefore, it is vital to add to the body of research on myocarditis and develop new therapeutic drugs. 
Dioscin is a type of saponin synthesized naturally by plants. Over the past few decades, the pharmacological effects of dioscin have been widely studied, demonstrating not only its obvious antitumor effects, but also its antiinflammatory properties, ability to regulate nervous system disorders, and other benefits $(2,3)$. It has been reported that dioscin can be used in the treatment of diabetes, cardiovascular disease, atherosclerosis, and other chronic diseases, and has good prospects for clinical application (4). For instance, dioscin reduces oxidative stress and inflammation to prevent coronary heart disease (5). However, the mechanism of anti-inflammation and antioxidation in myocarditis has not been elucidated.

Studies have shown that dioscin can reverse the significant increase of intracellular reactive oxygen species (ROS) levels in adriamycin-treated H9c2 cells (6). In view of these findings, this study aimed to explore the biological role and mechanism of dioscin in cardiomyocyte injury induced by lipopolysaccharide (LPS). A protocol was prepared before the study without registration. We found that dioscin reduced the damage of $\mathrm{H} 9 \mathrm{c} 2$ cells induced by LPS. The protective effect of dioscin on $\mathrm{H} 9 \mathrm{c} 2$ cells may be exerted through the inhibition of $\mathrm{Nrf} 2 / \mathrm{Keap} 1$ signal pathway. The findings suggest that dioscin may be a potential drug for the treatment of myocarditis.

We present the following article in accordance with the ARRIVE reporting checklist (available at https://dx.doi. org/10.21037/apm-21-1613).

\section{Methods}

\section{Use of animals}

The use of animals in the study was approved by The Shanxi Bethune Hospital Ethics Committee, and was in compliance with the institutional guidelines for the care and use of animals. The neonatal Sprague Dawley (SD) rats used to isolate primary cardiomyocytes were purchased from the experimental animal center of the Academy of Military Medical Sciences. Ten suckling SD rats on day 1 were selected.

\section{Isolation and culture of primary cardiomyocytes from neonatal rats}

Anesthetic was first injected into the caudal vein of SD rats, after which the atria and ventricles were removed, and the cardiac tissue was dissociated into single-cell suspension using collagenase type I (Sigma-Aldrich, St. Louis, MO, USA). The isolated cells were cultured in Dulbecco's Modified Eagle Medium (DMEM; Gibco, Waltham, MA, USA) containing $20 \%$ fetal bovine serum (FBS; HyClone, Logan, UT, USA) and antibiotics. The cells were incubated at $37^{\circ} \mathrm{C}$ in $5 \% \mathrm{CO}_{2}$ for 48 hours in 6-well plates at a density of $5 \times 10^{5} / \mathrm{mL}$.

\section{Cell treatment}

Cardiomyocytes were divided into the following groups: (I) control group, in which cardiomyocytes were cultured in a serum-free maintenance medium; (II) dioscin group, in which dioscin (Sigma-Aldrich; Merck KGaA; 50, 100, $200 \mathrm{ng} / \mathrm{mL}$ ) was used to treat cardiomyocytes for 0,12 , 24, or 48 hours; (III) dioscin + LPS group, in which cardiomyocytes were treated with dioscin $(50,100,100$, or $200 \mathrm{ng} / \mathrm{mL}$ ) for $0,12,24$, or 48 hours, stimulated with $1 \mu \mathrm{g} / \mathrm{mL}$ LPS for 4 hours, and incubated in humidified air containing $5 \% \mathrm{CO}_{2}$.

\section{Cell activity assay}

Cardiomyocytes in the logarithmic growth phase were cultured in 96-well plates with $100 \mu \mathrm{L}$ of cell culture per well (about $4 \times 10^{3} /$ well) and incubated for 24 hours at $37{ }^{\circ} \mathrm{C}$, with $5 \% \mathrm{CO}_{2}$ and $100 \%$ humidity. After being cultured for 24 hours, $50 \mu \mathrm{L}$ MTT $(5 \mathrm{~g} / \mathrm{L})$ was added and after incubation at $37^{\circ} \mathrm{C}$ for 4 hours, the supernatant was removed. Next, $150 \mu \mathrm{L}$ of dimethyl sulfoxide (DMSO) was added to each well, and the plates were shaken on a plate shaker. After the crystals dissolved, the optical density (OD) value of each well was detected at a wavelength of $570 \mathrm{~nm}$ with an enzyme labeling instrument.

\section{Determination of release of lactate debydrogenase (LDH)}

The culture medium of each group was collected after treatment. According to the manufacturer's instructions, LDH releases from the culture medium were measured using an LDH assay kit (Nanjing Jiancheng Bioengineering Institute, Nanjing, China).

\section{Flow cytometry}

Apoptosis was detected with Annexin V-FITC double staining. Twenty-four hours after transfection, the cells were digested by trypsin and then collected and inoculated 
in a 6-well plate. Cell density was adjusted to $2 \times 10^{6}$ cells per well and then cultured for 24 hours, after which the supernatant was discarded, the cells washed twice with phosphate-buffered saline (PBS), and then suspended in $1 \times$ binding buffer. After $5 \mu \mathrm{L}$ of Annexin V-FITC and $5 \mu \mathrm{L}$ of propidium iodide (PI) were added into the cell suspension, the apoptosis rate was detected by flow cytometry within 1 hour after incubation at room temperature for 15 minutes. The procedure was performed according to the instructions of the kit (BD Biosciences, San Jose, CA, USA).

\section{TUNEL staining}

The cells of each group were fixed with immunostaining fixative for 30 to 60 minutes and washed once with PBS. Immunostaining washing solution was added, and the cells incubated for 2 minutes in an ice bath. TUNEL detection solution $(50 \mu \mathrm{L})$ was added to the samples, which were incubated for 60 minutes at $37{ }^{\circ} \mathrm{C}$ and then washed with PBS 3 times. After anti-fluorescence quenching solution was added to the samples, fluorescence microscopy (excitation, 450-500 nm) was used to observe results. Five visual fields were randomly selected from each sample, and the apoptosis rate was calculated as follows: apoptosis rate $=$ apoptotic cells/total cells $\times 100 \%$.

\section{Enzyme-linked immunosorbent assay (ELISA)}

The cells were inoculated in a $24-$ well petri dish. After 24 hours of treatment, the culture supernatant was collected and the concentration of inflammatory cytokines interleukin-6 (IL-6), interleukin-1 $\beta$ (IL-1 $\beta$ ) and tumor necrosis factor- $\alpha(\mathrm{TNF}-\alpha)$ was measured by ELISA kit (Abcam, Shanghai, China).

\section{Determination of ROS}

The cells were inoculated in a 6-well culture plate with a density of $5 \times 10^{4}$ cells $/ \mathrm{mL}$. The cells were treated with deionized water $(50,100$, and $200 \mathrm{ng} / \mathrm{mL})$ for 24 hours, and then incubated with LPS for 24 hours. After removing the medium, DCFH-DA (dichloro-dihydro-fluorescein diacetate; $10.0 \mu \mathrm{m}, 1.5 \mathrm{~mL}$ ) was added to the wells and incubated at $37{ }^{\circ} \mathrm{C}$ for 25 minutes. Finally, the sample was observed at magnification using a fluorescence microscope (Olympus, Tokyo, Japan).

\section{Determination of oxidative stress markers superoxide dismutase (SOD), glutatbione, malondialdehyde}

The activity of SOD, glutathione (GSH), and the concentration of malondialdehyde (MDA) were detected according to the operation instructions of the kit. The hydroxylamine method was used for SOD, the colorimetric method for GSH, and the thiobarbituric acid (TBA) fluorescence method for MDA.

\section{Western blot}

Total protein was extracted and quantified using a bicinchoninic acid (BCA) protein concentration kit, and samples were prepared for SDS-PAGE (sodium dodecyl sulfate-polyacrylamide gel electrophoresis) with a bromophenol indicator. The selected gels were $5 \%$ concentrated gel and $10 \%$ separation gel, and $20 \mu \mathrm{L}$ protein samples were sampled. After electrophoresis, the protein was transferred to polyvinylidene fluoride (PVDF) membrane by electroporation under the condition of a $200 \mathrm{~mA}$ constant current. The PVDF membrane (Millipore, Bedford, MA, USA) was sealed with $10 \%$ skim milk powder solution for 2 hours, and the first antibody sAnti-Caspase 3 (1:1,000, ab32351), AntiBax (1:1,000, ab32503), Anti-Bcl2 (1:1,000, ab32124), Anti-Nrf2 (1:1,000, ab62352), Anti-HO-1 (1:1,000, ab52947), Anti-NQO1 (1:1,000, ab80588), Anti-GST (1:1,000, ab111947), Anti-GCLM (1:1,000, ab126704) and Anti-Keap1 (1:1,000, ab227888) were incubated at $4{ }^{\circ} \mathrm{C}$ overnight. TBST buffer of Tween-20 was used to wash the membrane for 3 times, 1 min each time. The membrane and the second antibody were incubated at room temperature for 2 hours, and the membrane was washed with TBST 3 times. Enhanced chemiluminescence (ECL) solution was used for color imaging of the protein. All the antibodies were purchased from abcam Company (Shanghai, China).

\section{Statistical analysis}

The data are expressed as the average \pm standard deviation of three independent experiments. SPSS software (version 17.0, IBM Corp., Armonk, NY, USA) was used for statistical analysis. Comparison between groups was analyzed by single-factor analysis of variance and Tukey posttest. A $\mathrm{P}$ value $<0.05$ was considered to be a statistically significant difference. 
A

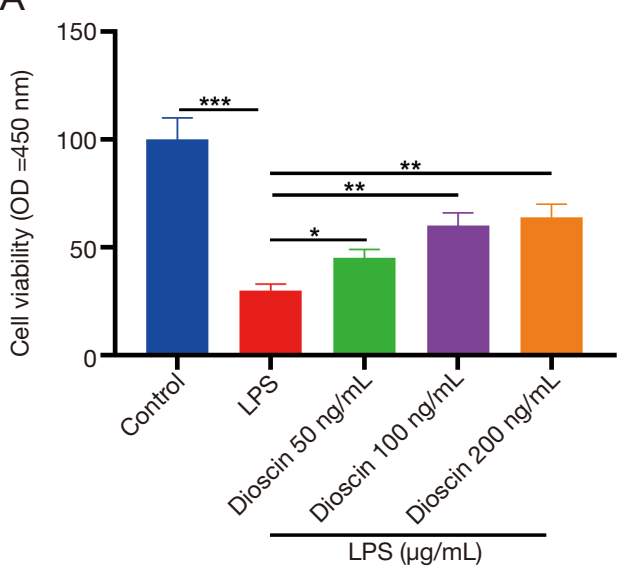

C

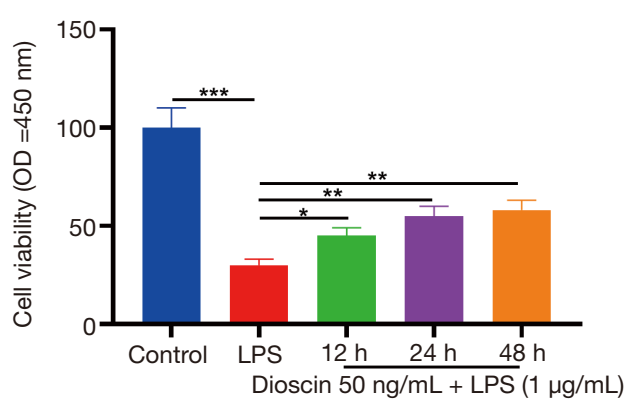

B

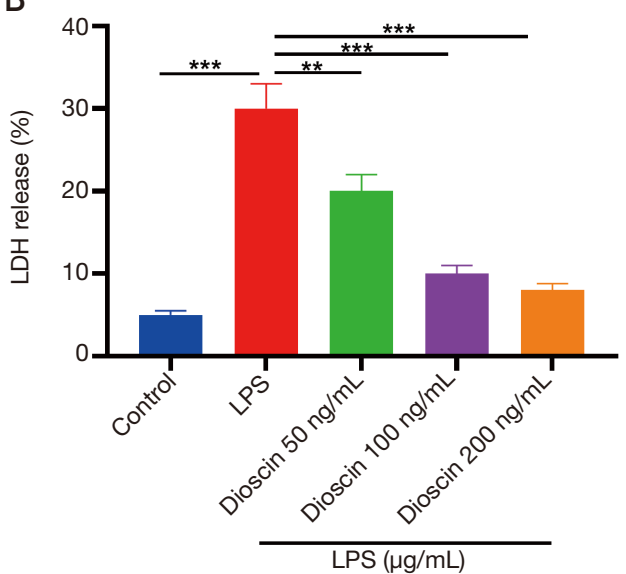

D

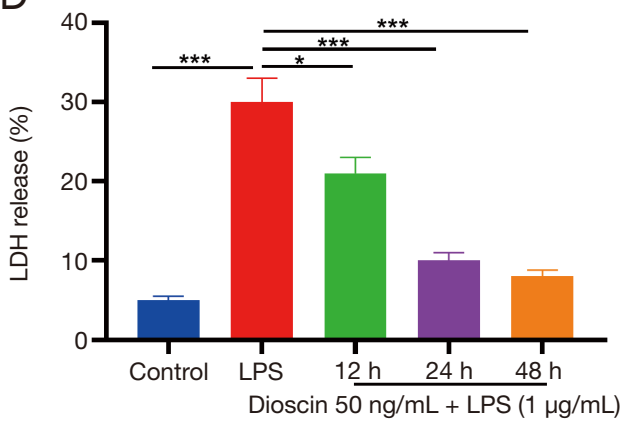

Figure 1 Dioscin enhanced the activity of cardiomyocytes induced by endotoxin. The effects of dioscin $(50,100$, and $200 \mathrm{ng} / \mathrm{mL})$ on LPSinduced cardiomyocytes. (A,B) MTT colorimetric assay and LDH release assay were used to detect the effect of dioscin on LPS-induced cardiomyocyte injury. LPS-induced cardiomyocytes were treated with dioscin (12, 24 and 48 h) at 24 hours; (C,D) MTT colorimetry and LDH release assay were used to detect the effect of dioscin on LPS-induced cardiomyocyte injury. LPS-induced cardiomyocytes were treated with $50 \mathrm{ng} / \mathrm{mL}$ of dioscin (at 12, 24, and 48 hours). *, $\mathrm{P}<0.05$; **, $\mathrm{P}<0.01$; *** $\mathrm{P}<0.001$. LDH, lactate dehydrogenase; LPS, liposaccharide.

\section{Results}

\section{Dioscin enhanced the activity of cardiomyocytes induced by LPS}

The effect of dioscin on endotoxin-induced cardiomyocyte injury was detected by colorimetry (MTT) and lactate dehydrogenase (LDH) release assay. Compared with the control group, LPS significantly decreased the survival rate of cardiomyocytes and increased the release of $\mathrm{LDH}$, while pretreatment with dioscin $(50,100$ and $200 \mathrm{ng} / \mathrm{mL}$ ) for 24 hours significantly increased the survival rate of cardiomyocytes stimulated by LPS and decreased the release of $\mathrm{LDH}$ in a concentration-dependent manner (Figure $1 A, 1 B ; \mathrm{P}<0.05$ ). Compared with the LPS group, cardiomyocytes pretreated with $50 \mathrm{ng} / \mathrm{mL}$ dioscin for different lengths of time (12, 24, and 48 hours) showed significantly increased cell viability and decreased $\mathrm{LDH}$ release in a time-dependent manner (Figure $1 C, 1 D ; \mathrm{P}<0.05$ ). The use of $50 \mathrm{ng} / \mathrm{mL}$ of dioscin for 24 hours was selected for follow-up experiments.

\section{Dioscin inbibited cardiomyocyte apoptosis and inflammation induced by LPS}

Subsequently, the effects of dioscin on cardiomyocyte apoptosis and inflammation induced by endotoxin were observed. The results of flow cytometry and TUNEL staining showed that apoptosis induced by LPS significantly increased compared with the control group, while apoptosis induced by dioscin significantly decreased after dioscin 
A

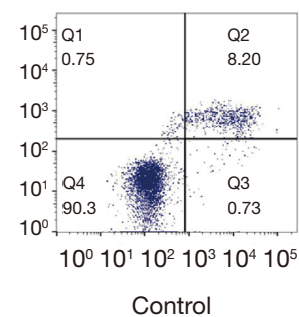

B
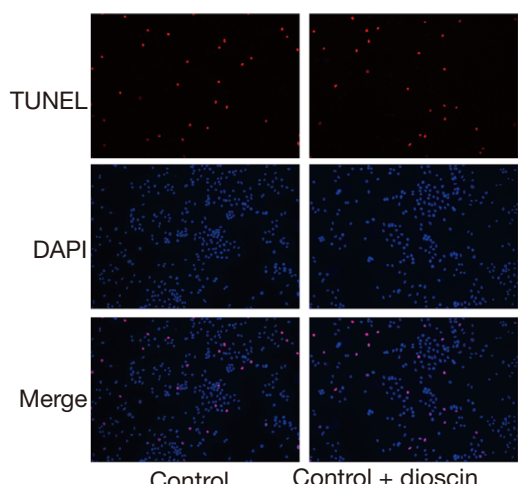

Control

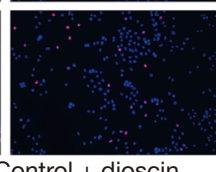

Control + dioscin $50 \mathrm{ng} / \mathrm{mL}$
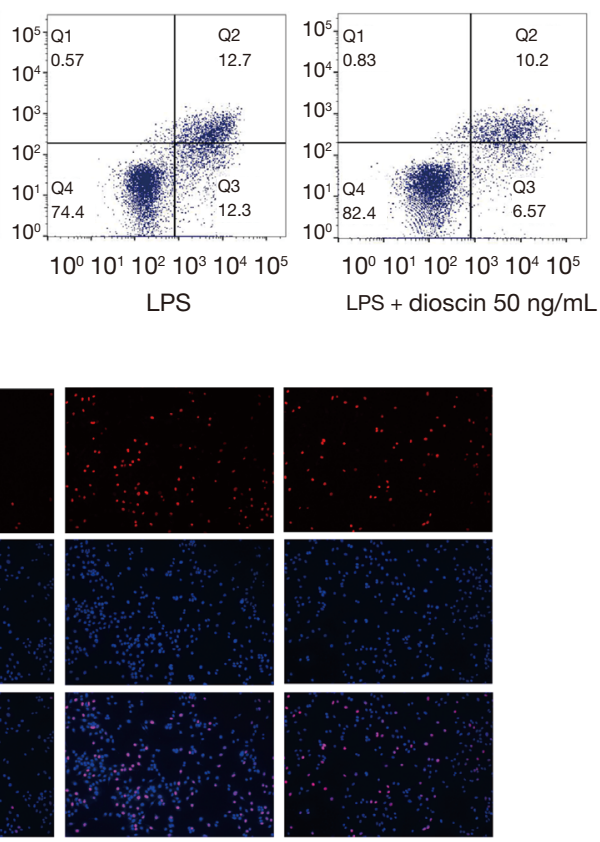

LPS
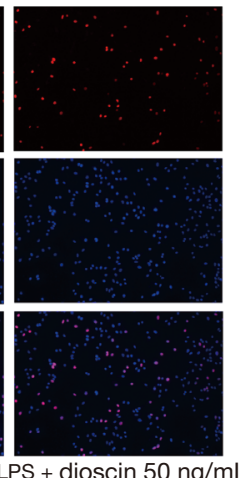

LPS + dioscin $50 \mathrm{ng} / \mathrm{mL}$

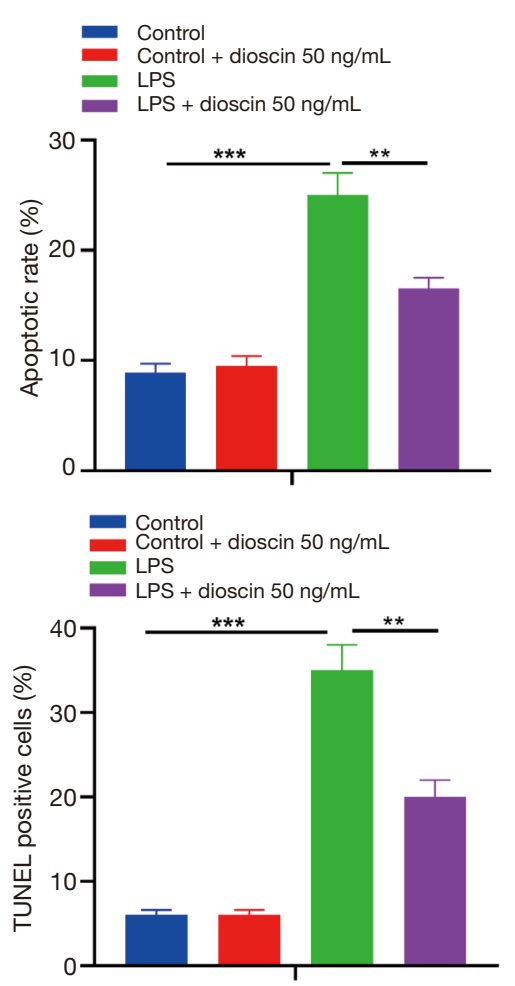

C
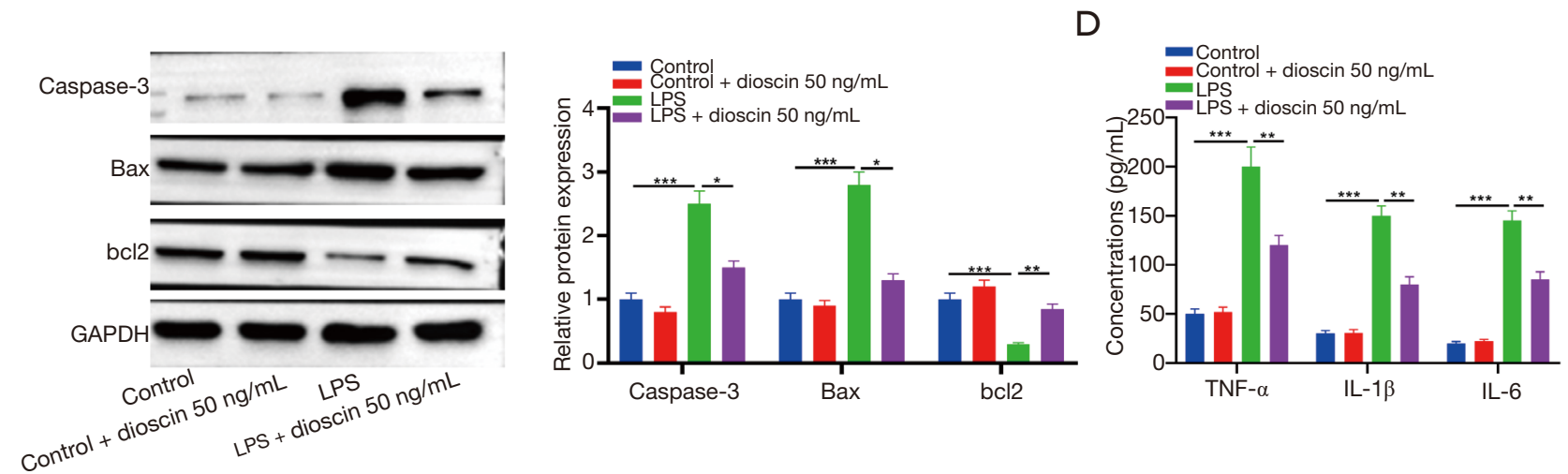

Figure 2 Dioscin inhibited cardiomyocyte apoptosis and inflammation induced by LPS. Cardiomyocytes induced by LPS were treated with $50 \mathrm{ng} / \mathrm{mL}$ of dioscin for 24 hours. (A,B) The level of apoptosis induced by LPS was detected by flow cytometry and TUNEL staining, the magnification was 100 times; (C) the levels of apoptosis-related proteins caspase-3, Bax and Bcl2 were detected by western blot; (D) the levels of proinflammatory cytokines TNF- $\alpha$, IL-1 $\beta$, and IL-6 were detected by ELISA. *, $\mathrm{P}<0.05 ;{ }^{* *}, \mathrm{P}<0.01$; *** $\mathrm{P}<0.001$. LPS, liposaccharide; TNF- $\alpha$, tumor necrosis factor alpha; IL, interleukin, ELISA, enzyme-linked immunosorbent assay.

pretreatment (Figure $2 A, 2 B ; \mathrm{P}<0.05$ ). In addition, dioscin pretreatment significantly reduced the increase of caspase- 3 and Bax activity and decreased Bcl-2 activity induced by LPS (Figure $2 C ; \mathrm{P}<0.05$ ). Compared with the control group, LPS significantly increased the expression of inflammatory cytokines TNF- $\alpha$, IL-1 $\beta$, and IL-6. However, dioscin preconditioning significantly decreased the expression of TNF- $\alpha$, IL- $\beta$, and IL-6 in LPS-treated cardiomyocytes (Figure $2 D ; \mathrm{P}<0.05$ ). The results show that dioscin reduced cardiomyocyte apoptosis and inflammation induced by LPS. 

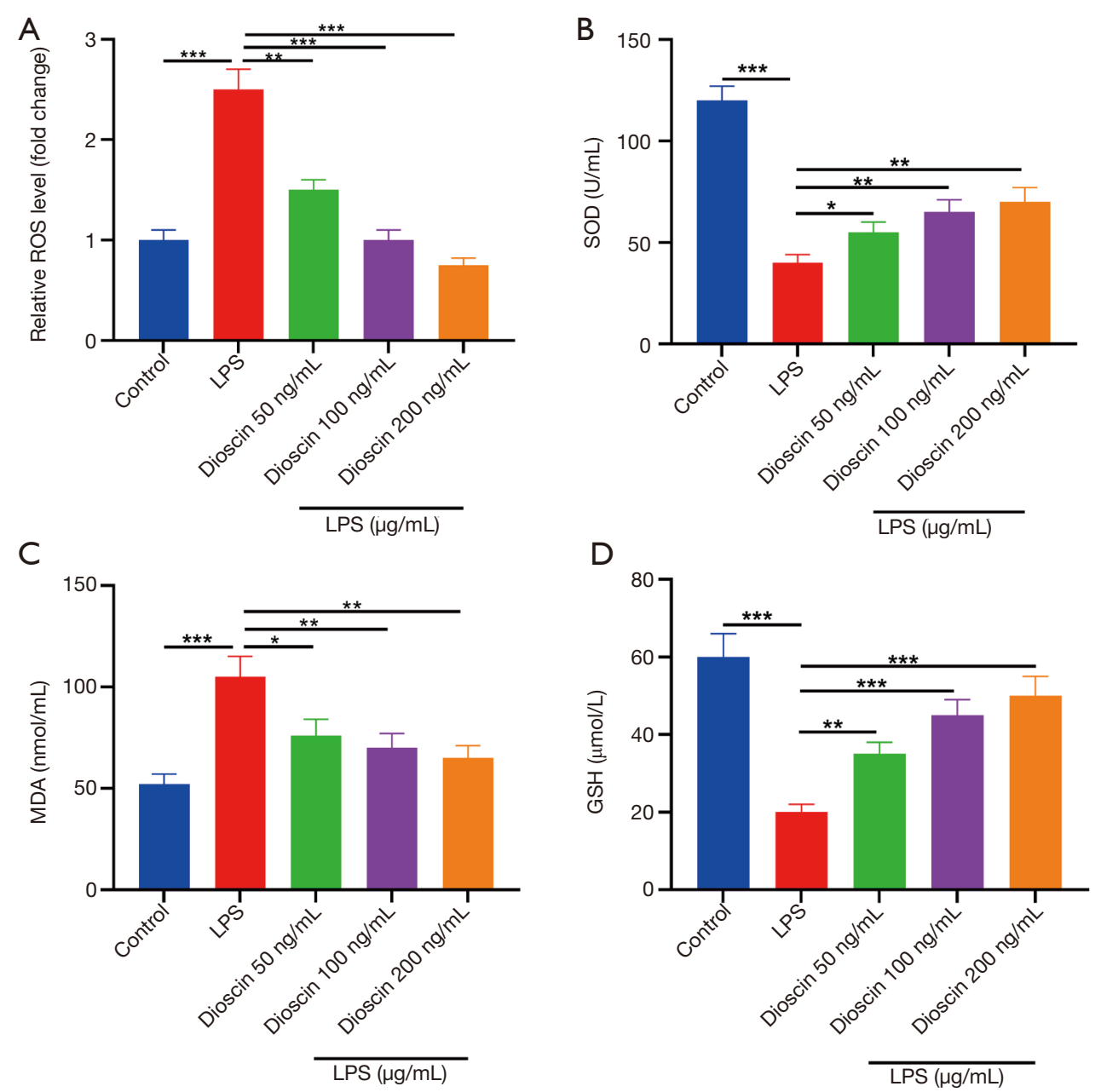

Figure 3 Dioscin inhibits LPS-induced oxidative stress injury of cardiomyocytes. Cardiomyocytes induced by LPS were treated with $50 \mathrm{ng} / \mathrm{mL}$ of dioscin for 24 hours. (A) The level of ROS was measured; (B-D) the activity of SOD, GSH, and the concentration of MDA were detected according to the operation instructions of the kit. * $\mathrm{P}<0.05$; **, $\mathrm{P}<0.01$; **, $\mathrm{P}<0.001$. LPS, liposaccharide; SOD, superoxide dismutase; GSH, glutathione; MDA, malondialdehyde; ROS, reactive oxygen species.

\section{Dioscin inbibited LPS-induced oxidative stress injury in cardiomyocytes}

The effect of dioscin on the oxidative stress of cardiomyocytes induced by LPS was observed. The analysis showed that the level of ROS in the LPS group was the highest, and that dioscin downregulated the level of ROS, and had a dose-dependent effect on the production of ROS (Figure $3 A ; \mathrm{P}<0.05$ ). Additionally, we measured the levels of SOD, MDA, and GSH in serum. Complementary to the results of ROS, the levels of serum SOD and GSH in the LPS group were the lowest, and the levels of SOD and GSH in serum increased in a dose-dependent manner after treatment with dioscin. The level of MDA in the LPS group was the highest, and the level of MDA in the dioscin group was significantly lower than that in the LPS group (Figure $3 B, 3 C ; \mathrm{P}<0.05$ ). Western blot showed that the expression of Nox1 and Nox2 in the LPS group was the highest, and the expression in the low-, middle-, and highdose groups was significantly lower than that in the LPS group (Figure 3D; $\mathrm{P}<0.05$ ).

Dioscin inbibited LPS-induced cardiomyocyte injury by inbibiting Nvf2-Keap1 oxidative stress patbway

As shown in Figure $4 A$ and $4 B$, the expressions of $\mathrm{Nrf2}$, HO-1, NQO1, GST, GCLM, and Keap1 in cardiomyocytes 
A

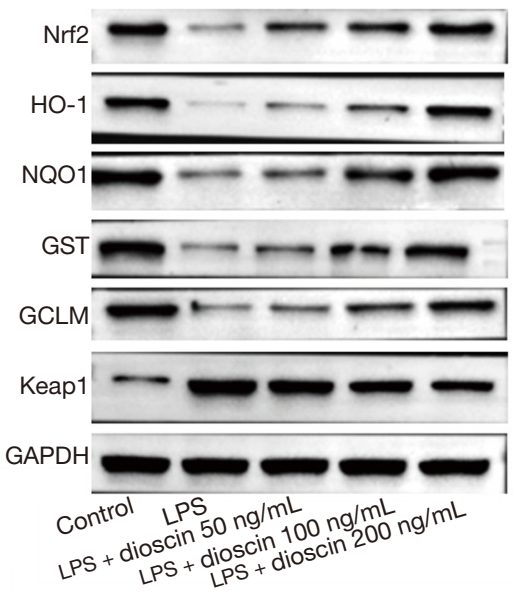

B

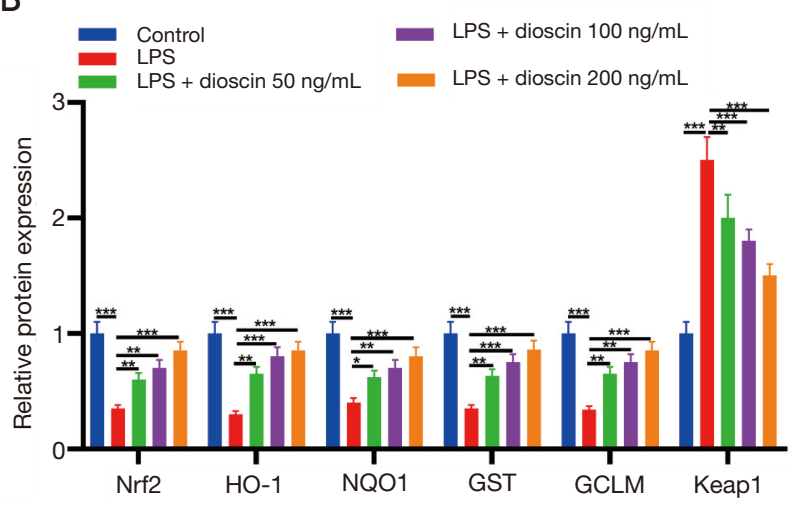

C

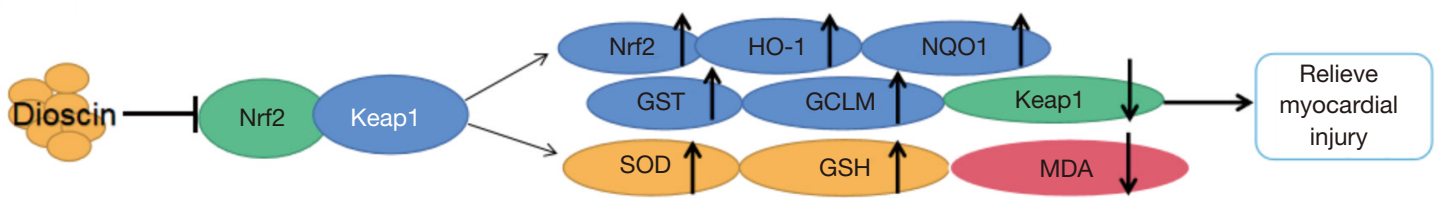

Figure 4 Dioscin inhibited Nrf2-Keap1-induced cardiomyocyte injury by inhibiting the LPS oxidative stress pathway. Cardiomyocytes treated with $50 \mathrm{ng} / \mathrm{mL}$ of dioscin were exposed to LPS for 24 hours. (A,B) The expressions of Nrf2, HO-1, NQO1, GST, GCLM, and Keap1 in cardiomyocytes were detected by western blot; (C) the mechanism map shows that dioscin inhibited Nrf2-Keap1-induced cardiomyocyte injury by inhibiting the LPS oxidative stress pathway. ${ }^{*}, \mathrm{P}<0.05 ;{ }^{* *}, \mathrm{P}<0.01 ;{ }^{* * *}, \mathrm{P}<0.001$. LPS, liposaccharide.

were detected by western blot. The results showed that the expression levels of Nrf2, HO-1, NQO1, GST, and GCLM in cardiomyocytes induced by LPS significantly decreased, while the level of Keap1 was upregulated. Compared with the LPS group, dioscin significantly increased the expression levels of Nrf2, HO-1, NQO1, GST, and GCLM, and decreased the expression level of Keap1. The results show that dioscin alleviated LPS-induced myocardial injury by regulating the Nrf2-Keap1 oxidative stress pathway (Figure 4C).

\section{Discussion}

Myocarditis is a serious life-threatening and crippling cardiovascular disease. It is associated with the infiltration of a large number of inflammatory cells and the release of proinflammatory cytokines, which can directly damage the contractile and diastolic function of myocardial cells, causing changes in cardiac electrical activity and conduction disorders $(7,8)$. In addition to inflammation, NADPH (nicotinamide adenine dinucleotide phosphate) oxidase produces free radicals during phagocytosis of myocarditis to meet increased oxygen consumption (9). In this study, it was found that the survival rate of cardiomyocytes stimulated by LPS decreased significantly and the release of LDH increased significantly, suggesting that LPS induced cardiomyocyte injury. Excessive production of inflammatory mediators is considered to be one of the main potential mechanisms in LPS-induced myocardial injury (10). Inflammatory mediators can lead to the loss of activation of caspase- 3 , which eventually results in cardiomyocyte apoptosis $(11,12)$. Cardiomyocyte apoptosis has been confirmed to be associated with myocardial dysfunction (13). One study found the levels of TNF- $\alpha$ and IL-1 $\beta$ in myocardium of LPS-injured mice to be increased (14). Consistent with previous studies, this study found that LPS upregulated the levels of proinflammatory cytokines, including TNF- $\alpha$, IL- 6 , and IL- $1 \beta$, inhibiting cell activity and promoting cell apoptosis.

Dioscin is an effective therapeutic component in many kinds of traditional Chinese medicine. It is abundant several in plants, such as Dioscorea, Liliaceae, Caryophyllaceae, and others. Studies have shown that dioscin can contribute to many biological processes, including cell processes, 
metabolic processes, biological regulation, stress responses, signal pathways, and cell proliferation (15). Pharmacological studies have shown that dioscin has antitumor and antifungal effects $(16,17)$. A large number of previous studies have shown that dioscin has a beneficial effect on hepatic fibrosis, renal injury, and cerebral ischemia/ reperfusion injury $(8,18,19)$. It is worth nothing that an increasing number of studies have focused on the protective effects of dioscin for myocardial injury. It has been found, for instance, that dioscin plays a protective role in adriamycin-induced cardiotoxicity by inhibiting the Nrf2Keap1 signaling pathway (20). Previous studies have also shown that dioscin can prevent mitochondrial apoptosis and reduce oxidative stress in cardiac H9c2 cells (21), suggesting that dioscin protects injured $\mathrm{H} 9 \mathrm{c} 2$ cells against oxidative stress. Furthermore, research has begun to show that oxidative stress is involved in the biological process of dioscin. Qiao et al. demonstrated that dioscin alleviates renal injury by regulating SIRT3-mediated oxidative stress, renal fibrosis, lipid metabolism, and inflammation (3). Meanwhile, dioscin has been shown to alleviate liver and kidney injury induced by methotrexate through oxidative stress (7). Consistent with the above studies, the experiments of this study showed that dioscin promoted the growth of primary cardiomyocytes of neonatal rats induced by LPS and inhibited apoptosis in a concentration-dependent manner, suggesting that dioscin can inhibit the growth of $\mathrm{H} 9 \mathrm{c} 2$ cells induced by LPS.

Nrf2-Keap1 is the key signal pathway of antioxidant injury. Nrf2 is an important regulator of cellular oxidative stress, which increases the antioxidant capacity of cells by promoting downstream-related antioxidant enzymes. Keap1 is a regulatory factor of $\mathrm{Nrf2}$, which couples with $\mathrm{Nrf} 2$ to form a complex to inhibit the activity of Nrf2. When cells are stimulated by oxidative stress, Keap1 is uncoupled from $\mathrm{Nrf2}$, and the steady-state $\mathrm{Nrf} 2$ translocates to the nucleus. Combined with the antioxidant response element (ARE), Nrf2 initiates the expression of ARE-regulated detoxification enzymes and antioxidant enzymes, such as heme oxygenase 1 (HO-1), SOD, and GSH-PX (22). It has been found that Nrf2-Keap1 pathway plays an important role in myocardial injury (23), and studies have shown that baicalein protects cardiomyocytes from oxidative stress-induced cell injury through Nrf2-Keap1 pathway (24). Additionally, xanthine has a cytoprotective effect on oxidized low-density lipoprotein-induced cell injury through the activation of Nrf2-ARE-mediated antioxidant stress (25). Dioscin also plays a protective role in adriamycin-induced cardiotoxicity by inhibiting the Nrf2-Keap1 signal pathway (20). In line with the above studies, it was found that dioscin significantly increased the expression levels of Nrf2, HO-1, NQO1, GST, and GCLM, and decreased the expression level of Keap1 in LPS-induced H9c2 cells. This suggests that dioscin can regulate the progression of myocarditis by regulating the Nrf2-Keap1 axis.

In summary, dioscin can protect primary cardiomyocytes of neonatal rats from LPS-induced inflammatory injury by improving cell viability, reducing apoptosis, and inhibiting the production of inflammatory cytokines and oxidative stress factors. Dioscin may play a protective role by inhibiting Nrf2-Keap1 pathway. These results provide a basis for dioscin as a potential drug for the treatment of myocarditis. However, this study has limitations. In order to further verify the effect of dioscin on myocarditis, clinical sample experiments and in vivo experiments need to be carried out. To conclude, this study found that dioscin has a protective effect against LPS-induced injury to H9c2 cells, which may provide a scientific basis for the treatment of myocarditis and the development of safe drugs.

\section{Acknowledgments}

Funding: None.

\section{Footnote}

Reporting Checklist: The authors have completed the ARRIVE reporting checklist. Available at https://dx.doi. org/10.21037/apm-21-1613

Data Sharing Statement: Available at https://dx.doi. org/10.21037/apm-21-1613

Conflicts of Interest: All authors have completed the ICMJE uniform disclosure form (available at https://dx.doi. org/10.21037/apm-21-1613). The authors have no conflicts of interest to declare.

Ethical Statement: The authors are accountable for all aspects of the work in ensuring that questions related to the accuracy or integrity of any part of the work are appropriately investigated and resolved. Animal experiments were approved by The Shanxi Bethune Hospital Ethics 
Committee, and were performed in compliance with the institutional guidelines for the care and use of animals.

Open Access Statement: This is an Open Access article distributed in accordance with the Creative Commons Attribution-NonCommercial-NoDerivs 4.0 International License (CC BY-NC-ND 4.0), which permits the noncommercial replication and distribution of the article with the strict proviso that no changes or edits are made and the original work is properly cited (including links to both the formal publication through the relevant DOI and the license). See: https://creativecommons.org/licenses/by-nc-nd/4.0/.

\section{References}

1. Ammirati E, Veronese G, Cipriani M, et al. Acute and Fulminant Myocarditis: a Pragmatic Clinical Approach to Diagnosis and Treatment. Curr Cardiol Rep 2018;20:114.

2. Tao X, Yin L, Xu L, et al. Dioscin: A diverse acting natural compound with therapeutic potential in metabolic diseases, cancer, inflammation and infections. Pharmacol Res 2018;137:259-69.

3. Qiao $\mathrm{Y}, \mathrm{Xu} \mathrm{L}$, Tao X, et al. Protective effects of dioscin against fructose-induced renal damage via adjusting Sirt3mediated oxidative stress, fibrosis, lipid metabolism and inflammation. Toxicol Lett 2018;284:37-45.

4. Cheng J, Sun C, Zhang J, et al. The Protective Effects of Preconditioning With Dioscin on Myocardial Ischemia/ Reperfusion-Induced Ventricular Arrhythmias by Increasing Connexin 43 Expression in Rats. J Cardiovasc Pharmacol Ther 2019;24:262-8.

5. Yang B, Xu B, Zhao H, et al. Dioscin protects against coronary heart disease by reducing oxidative stress and inflammation via Sirt1/Nrf2 and p38 MAPK pathways. Mol Med Rep 2018;18:973-80.

6. Zhao L, Tao X, Qi Y, et al. Corrigendum to 'Protective effect of dioscin against doxorubicin-induced cardiotoxicity via adjusting microRNA-140-5p-mediated myocardial oxidative stress' Redox Biol., 2018, 16: 189-198. Redox Biol 2019;26:101303.

7. Frey T, Arain N. Pediatric Viral Myocarditis - A Review. S D Med 2018;71:29-34.

8. Crişan S, Tint D, Petrescu L. Therapeutic Advances in Emergency Cardiology: A Focus on Acute Myocarditis. Am J Ther 2019;26:e294-300.

9. Raucci A, Di Maggio S, Scavello F, et al. The Janus face of HMGB1 in heart disease: a necessary update. Cell Mol Life Sci 2019;76:211-29.
10. Li M, Ye J, Zhao G, et al. Gas6 attenuates lipopolysaccharide-induced TNF- $\alpha$ expression and apoptosis in $\mathrm{H} 9 \mathrm{C} 2$ cells through $\mathrm{NF}-\kappa \mathrm{B}$ and MAPK inhibition via the Axl/PI3K/Akt pathway. Int J Mol Med 2019;44:982-94.

11. Fan JH, Feng GG, Huang L, et al. Naofen promotes TNF- $\alpha$-mediated apoptosis of hepatocytes by activating caspase-3 in lipopolysaccharide-treated rats. World J Gastroenterol 2014;20:4963-71.

12. Sangaran PG, Ibrahim ZA, Chik Z, et al. LPS Preconditioning Attenuates Apoptosis Mechanism by

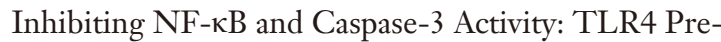
activation in the Signaling Pathway of LPS-Induced Neuroprotection. Mol Neurobiol 2021;58:2407-22.

13. Gao G, Chen W, Yan M, et al. Rapamycin regulates the balance between cardiomyocyte apoptosis and autophagy in chronic heart failure by inhibiting mTOR signaling. Int J Mol Med 2020;45:195-209.

14. Ye T, Zhang C, Wu G, et al. Pinocembrin attenuates autonomic dysfunction and atrial fibrillation susceptibility via inhibition of the $\mathrm{NF}-\kappa \mathrm{B} / \mathrm{TNF}-\alpha$ pathway in a rat model of myocardial infarction. Int Immunopharmacol 2019;77:105926.

15. Du S, Li C, Lu Y, et al. Dioscin Alleviates Crystalline Silica-Induced Pulmonary Inflammation and Fibrosis through Promoting Alveolar Macrophage Autophagy. Theranostics 2019;9:1878-92.

16. Cui L, Yang G, Ye J, et al. Dioscin elicits anti-tumour immunity by inhibiting macrophage M2 polarization via JNK and STAT3 pathways in lung cancer. J Cell Mol Med 2020;24:9217-30.

17. Ran X, Yan Z, Yang Y, et al. Dioscin Improves Pyroptosis in LPS-Induced Mice Mastitis by Activating AMPK/Nrf2 and Inhibiting the NF- $\kappa$ B Signaling Pathway. Oxid Med Cell Longev 2020;2020:8845521.

18. Xu L, Yin L, Tao X, et al. Dioscin, a potent ITGA5 inhibitor, reduces the synthesis of collagen against liver fibrosis: Insights from SILAC-based proteomics analysis. Food Chem Toxicol 2017;107:318-28.

19. Qi M, Yin L, Xu L, et al. Dioscin alleviates lipopolysaccharide-induced inflammatory kidney injury via the microRNA let-7i/TLR4/MyD88 signaling pathway. Pharmacol Res 2016;111:509-22.

20. Zhao L, Tao X, Qi Y, et al. Protective effect of dioscin against doxorubicin-induced cardiotoxicity via adjusting microRNA-140-5p-mediated myocardial oxidative stress. Redox Biol 2018;16:189-98.

21. Qin J, Kang Y, Xu Z, et al. Dioscin prevents the 
mitochondrial apoptosis and attenuates oxidative stress in cardiac H9c2 cells. Drug Res (Stuttg) 2014;64:47-52.

22. Zhuang $\mathrm{Y}, \mathrm{Wu} \mathrm{H}$, Wang $\mathrm{X}$, et al. Resveratrol Attenuates Oxidative Stress-Induced Intestinal Barrier Injury through PI3K/Akt-Mediated Nrf2 Signaling Pathway. Oxid Med Cell Longev 2019;2019:7591840.

23. Xiao X, Lu Z, Lin V, et al. MicroRNA miR-24-3p Reduces Apoptosis and Regulates Keap1-Nrf2 Pathway in Mouse Cardiomyocytes Responding to Ischemia/Reperfusion

Cite this article as: $\mathrm{Xu} \mathrm{Z,} \mathrm{Li} \mathrm{X,} \mathrm{Li} \mathrm{X,} \mathrm{Gao} \mathrm{Y,} \mathrm{Mi} \mathrm{X.} \mathrm{Dioscin}$ attenuates lipopolysaccharide-induced inflammatory myocardial injury through oxidative stress-related pathway. Ann Palliat Med 2021;10(8):8827-8836. doi: 10.21037/apm-21-1613
Injury. Oxid Med Cell Longev 2018;2018:7042105.

24. Cui G, Luk SC, Li RA, et al. Cytoprotection of baicalein against oxidative stress-induced cardiomyocytes injury through the Nrf2/Keap1 pathway. J Cardiovasc Pharmacol 2015;65:39-46.

25. Yan R, Yan J, Chen X, et al. Xanthoangelol Prevents OxLDL-Induced Endothelial Cell Injury by Activating Nrf2/ ARE Signaling. J Cardiovasc Pharmacol 2019;74:162-71. 